\title{
Editorial: Special Issue on Resource and Mobility Management and Cross-Layer Design for the Support of Multimedia Services in Heterogeneous Emerging Wireless Networks
}

\author{
Tasos Dagiuklas • Christos Politis • \\ Jonathan Rodriguez
}

Published online: 13 November 2008

(C) The Author(s) 2008. This article is published with open access at Springerlink.com

The Future Networks' vision towards a diverse wireless networking world, considers the support of any service, at any time delivered in a transparent optimized way. The demand for multimedia services delivered "anywhere and anyhow" has increased tremendously over the last few years leading to the evolution of the wireless industry towards all-IP networking. The research effort for the interworking heterogeneous technologies (e.g. WLANs, ad-hoc, wireless mesh, 4G, WiMAx, LTE etc.) amplifies the need to evaluate the conditions and restrictions to deliver these services. The QoS of multimedia services such as IP telephony, video-on-demand and mobile video will depend greatly on the ability to minimize the impact of the handover, hence traffic redirection in a seamless and secure transparent manner. Furthermore, Cross-layer and Cross-system optimization solutions will also be the enablers to deliver multimedia services over heterogeneous networks in a cost-effective manner.

The main objectives in this special issue are to address novel research results in the areas of resource and mobility management protocols and cross-layer techniques to facilitate effective cooperation among heterogeneous wireless networks thus enabling seamless service mobility to the 'end-user' in an optimized way.

The following papers have been included at this special issue:

The first paper is entitled 'Scalability Issues in Ad-Hoc Networks: Metrical Routing Versus Table-Driven Routing' by Yosi Ben-Asher, Sharoni Feldman and Moran Feldman. They study the scalability of routing schemes for ad-hoc networks using explicit clustering. They compare an advanced version of the Ad Hoc Distance Vector Algorithm (AODV) by incorporating the Metrical Routing Algorithm (MRA) that possesses the natural clustering property.

The second paper entitled 'Timely Effective Handover Mechanism in Heterogeneous Wireless Networks' by Sang-Jo Yoo, David Cypher, Nada Golmie propose a new predictive handover framework in order to achieve seamless handover in diverse wireless environments. Their approach uses the neighbor network information to generate timely the link triggers

T. Dagiuklas $(\bowtie) \cdot C$. Politis · J. Rodriguez

Department of Telecommunication Systems \& Networks Technological, Educational Institute of Mesolonghi, Nafpaktos, Greece

e-mail: fu@cs.uni-goettingen.de 
so that the required handover procedures can appropriately finish before the current link goes down.

The third paper entitled 'Trust-Assisted Handover Approach in Hybrid Wireless Networks" by Mo Li, Kumbesan Sandrasegaran and Tracy Tung present a trust-assisted handover scheme to resolve the multi-operator problem caused by the coexistence of a large number of network operators in Next Generation Networks. The proposed scheme can support more efficient and reliable handover than the existing handover algorithms using two separated phases: neighbor trust information retrieval and trust-assisted handover decision making.

The forth paper entitled "A Handover Prediction Model and its Application to Link Layer Triggers for Fast Handover" by Jaesung Park and Yujin Lim, considers Cross layer handover schemes in order to provide seamless services to users on the move. They propose a mobility model that does not assume any radio propagation environments and movement patterns of a mobile node. Through statistical analysis of the received signal strength index (RSSI) datasets measured in various radio propagation environments, they validate that an adaptive autoregressive process can be used as a handover prediction model.

The fifth paper entitled "Utility based Short-term Throughput Driven Scheduling Approach for Efficient Resource Allocation in CDMA Wireless Networks" by Timotheos Kastrinogiannis and Symeon Papavassiliou addresses the problem of efficient recourse allocation in CDMA wireless networks supporting multimedia services with various and often diverse QoS prerequisites. They propose and develop a scheduling policy for efficiently supporting heterogeneous services that include delay-tolerant non-real-time and delay-sensitive real-time services, over a wireless CDMA system under a common utility based framework.

The sixth paper entitled "Performance Metrics for IEEE 802.21 Media Independent Handover (MIH) Signaling” by David Griffith, Richard Rouil, and Nada Golmie, has developed a mathematic model that reflects to the characteristics of Media Independent Handover between a Mobile Node and a remote Mobility Manager. Their model provides expressions for the average amount of overhead associated with MIH message exchanges due to retransmissions either by the MIH signaling entities or by the transport-layer protocol.

The seventh paper entitled "Meeting Interactivity Requirements an Experimental Study" by Vittorio Ghini, Giorgia Lodi, Stefano Cacciaguerra, Fabio Panzieri describes the design and development of a session-layer load balancing mechanism and its deployment in a mobile surveillance system named Mobile E-Witness (MEW). The load balancing mechanism allows MEW to effectively meet interactivity requirements and it is based on an "early retransmission" technique that exploits the overall bandwidth provided by a number of heterogeneous (broadband and metropolitan area) wireless adapters incorporated in MEW.

The eight paper "A Robust Seamless Handover scheme for the Support of Multimedia Services in Heterogeneous Emerging Wireless Networks" by Hayoung Oh, Kibaek Yoo, Jongkeun $\mathrm{Na}$ and Chong-kwon Kim, proposes a robust seamless handover scheme for the multimedia services in heterogeneous emerging wireless networks. The proposed scheme reduces the handover latency and handover initiation time when handover may fail through the management of tentative Care-of Addresses (CoAs) that does not require Duplicate Address Detection (DAD).

The ninth paper "Handover Management in Enhanced MIH Framework for Heterogeneous Wireless Networks Environment" by Ying Wang, Ping Zhang, Yun Zhou, Jun Yuan, Fang Liu, Gen Li, an Enhanced Media Independent Handover (EMIH) framework is proposed by integrating more information from application layers, user context and network context. A new approach is proposed for network selection in heterogeneous wireless network environment. At first, the candidate networks are ranked by each decision factor and 
multiple decision factor based ranking lists are obtained accordingly. These ranking lists are then aggregated into a new ranking list according to a certain criterion and the top one network of new ranking list is finally considered as favorite network.

The tenth paper entitled "A DVB/IP QoS aware Backhaul Networking Environment" by George Mastorakis, Evangelos Pallis and George Kormentzas, they propose DVB/IP backhaul networking environment that enables users to access triple-play IP services at a guaranteed end-to-end QoS level. The capability of the proposed QoS aware DVB/IP backhaul networking environment is validated through experimental tests.

The eleventh paper entitled "A cross-system approach for multimedia services with IP multicast in 4G networks" by Diogo Gomes, Rui Aguiar, Susana Sargento, propose a new architecture that builds itself on the synergies created by a heterogeneous network made of existing delivering technologies, such as 3GPP/MBMS and DVB, where services can be delivered to end-users in the most appropriate way for end-users and operators alike.

The twelfth paper entitled "A Cross-Layer Approach for Improving TCP Performance in Mobile Environments" by Deguang Le, Xiaoming Fu, Dieter Hogrefe, proposes a new crosslayer approach, by introducing a mobility detection element in the network layer which interacts with the transport layer to optimize TCP operations by preserving the end-to-end semantics of TCP.

Open Access This article is distributed under the terms of the Creative Commons Attribution Noncommercial License which permits any noncommercial use, distribution, and reproduction in any medium, provided the original author(s) and source are credited.

\section{Author Biographies}

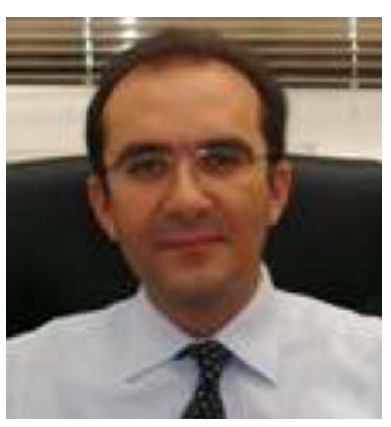

Tasos Dagiuklas was born in Patras, Greece. He received the Engineering Degree from the University of Patras-Greece in 1989, the M.Sc. from the University of Manchester-UK in 1991 and the Ph.D. from the University of Essex-UK in 1995, all in Electrical Engineering. Currently, he is employed as Assistant Professor at the Department. of Telecommunications and Network Systems, Technical Educational Institute of Mesolonghi. He is also Senior Research Associate within the Wireless Telecommunications Laboratory of the Electrical and Computer Engineering Department at the University of Patras, Greece. Past Positions include teaching Staff at the University of Aegean, Department of Information and Communications Systems Engineering, Greece, senior posts at INTRACOM and OTE, Greece. During the summer of 1995, he obtained a short fellowship sponsorship from British Telcom Labs, Ipswich, UK. He has been involved in several EC R\&D Research Projects under FP5, FP6 and FP7 research frameworks, in the fields of All-IP network and next generation services. Currently, he is the Technical Manager of the FP7-ICT-PEACE project. He was the Conference General Chair of the international conference, Mobile Multimedia 2007 (Mobimedia 2007) and Technical Co-Chair of MMNS Conference of MANWEEK 2007 conference and he is the Workshop Chair for Mobimedia 2009. His research interests include All-IP Networks, systems beyond $3 \mathrm{G}$ and converged multimedia services over fixed-mobile networks. Dr Dagiuklas ha published more than 70 papers at international journals and conferences in the above fields. He is a member of IEEE and Technical Chamber of Greece. 


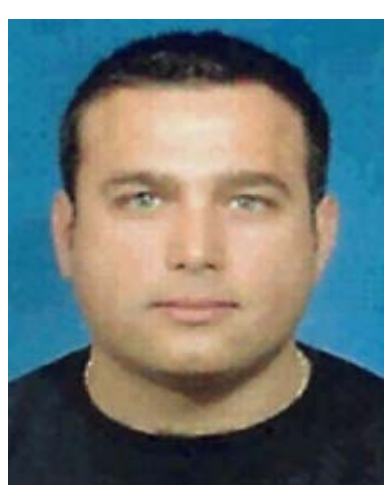

Christos Politis is Assistant Professor (Senior Lecturer) at Kingston University, London, UK, Faculty of Computing, Information Systems and Mathematics (CISM). There he is leading a research group on Wireless Multimedia Networking (WMN). Prior to this he was the Research and Development (R\&D) project manager at Ofcom, the UK Regulator and Competition Authority. There he managed a number of projects across a wide range of areas including cognitive radio, polite protocols, radar, fixed wireless and mobile technologies. He was also responsible for the Ofcom R\&D budget. Christos's previous positions include telecommunications engineer with INTRACOM SA in Athens and for many years he was a senior researcher at the Centre for Communication Systems Research (CCSR) at the University of Surrey, UK. He is/ has been involved with several EU (Information Society Technologies), national and international projects, and was the project manager of the IST UNITE. Christos is a patent holder, and has published more than 50 papers at international journals and conferences and chapters in two books. Christos holds a Ph.D. and M.Sc. from the University of Surrey and a B.Eng. from the Technical University of Athens, Greece. He is a member of IEEE and Technical Chamber of Greece.

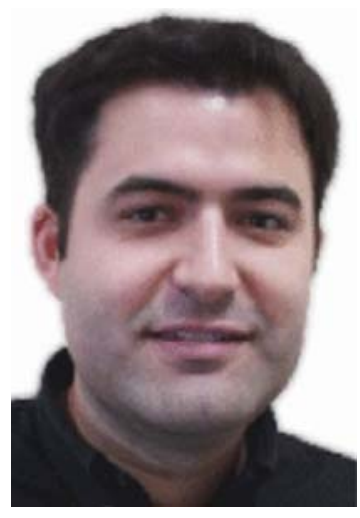

Jonathan Rodriguez received his Masters degree in Electronic and Electrical engineering and Ph.D. from the University of Surrey, in 1998 and 2004 respectively. In 2002, he became a Research Fellow at the Centre for Communication Systems Research at Surrey. He has been actively leading system level technical activities in the IST MATRICE, 4MORE and MAGNET projects. Since 2005, he is a Senior Researcher at the Instituto de Telecomunicações, Pólo de Aveiro, and in 2008 became an invited professor at the University of Aveiro. He is author of several conference and journal publications, and has carried out consultancy for major manufacturers participating in DVB-T and HS-UPA standardization. His research interests include Radio Access Networks for legacy and beyond 3G systems with specific emphasis on Radio Resource Management, Cross-Layer design, and signal processing techniques. 